\section{Tiempo de cirugía efectiva de la tercera molar mandibular y factores de dificultad}

\author{
Effective time of surgery of the third mandibular molar and \\ difficulty factors
}

\begin{abstract}
Resumen
Objetivo: Determinar la relación existente entre el tiempo de cirugía efectiva y los factores de dificultad en la extracción del tercer molar mandibular. Materiales y Métodos: El estudio fue descriptivo. Se evaluaron 30 extracciones realizadas por un cirujano maxilofacial. Los parámetros tomados fueron: espacio disponible, profundidad, relación espacial, tamaño del folículo, integridad de hueso y mucosa y forma de raíces. Se calculó las frecuencias de las variables. Se continúo con un análisis bivariado a través del uso de coeficientes de correlación de Pearson, relacionando el tiempo con todas las variables. Resultados: El tiempo promedio fue de $9.63 \mathrm{~min}$. Se encontró con mayor frecuencia: clase II, nivel B, posición mesioangular, tamańo de folículo entre 0 a $1 \mathrm{~mm}$, cubierto totalmente por mucosa y parcialmente por hueso y la forma más frecuente de las raíces fueron $2 / 3$ fusionadas y más de $2 / 3$ separadas. Conclusiones: El tiempo de cirugía efectiva guarda relación significativa con el tamańo del folículo, la integridad de hueso y mucosa y la profundidad. El espacio disponible, relación espacial y la forma de las raíces no fueron significativas. Existe una relación directa entre el tiempo de cirugía efectiva y la edad.
\end{abstract}

Palabras clave: Cirugía Bucal/Tercer Molar/ tiempo de cirugía efectiva

\begin{abstract}
Objective: To determine the relationship between effective time of surgery, with the factors of difficulty for the extraction of mandibular third molar. Materials and Methods: The study was descriptive. 30 extractions were evaluated by a maxillofacial surgeon. The parameters taken were: space, depth, spatial relationships, size of the follicle, and the integrity of bone and y mucosa, and form roots. We calculated the frequencies of the variables and covariates. It continues with a bivariate analysis using Pearson correlation coefficients, relating the time with all the variables. Results: The average time of third molar extraction was $9.63 \mathrm{~min}$. It was found more frequently Class II, Level B, mesioangular position, size of follicle from $0-1 \mathrm{~mm}$., completely covered by mucosa and partly by bone and the most common form of the roots were $2 / 3$ combined and more than $2 / 3$ apart. Conclusions: effective time of surgery is related significantly with size of the follicle, the integrity of bone and mucosa and depth. The available space, spatial relationships and form roots were not significant. There is a direct relationship between the effective time of surgery and age. Key words: Oral Surgery / Third Molar / effective time of surgery.
\end{abstract}

Artículo Original

\section{María Vásquez Vásquez'1, Arturo Rodríguez Flores², José Cornejo Salazar ${ }^{2}$, Andy Sotelo Ortiz $^{3}$}

\footnotetext{
Cirujana Dentista. Universidad Nacional Mayor de San Marcos.

${ }^{2}$ Docente del Departamento Académico Médico Quirúrgico. Universidad Nacional Mayor de San Marcos.

3 Residente de segundo año de Cirugía Oral y Maxilofacial. Universidad Nacional Mayor de San Marcos.
}

Correspondencia:

María Vásquez Vásquez

Dirección: Jr. Aurelio Miroquezada 539. Ingenieria. SMP

Teléfono: 997204023

Correo electrónico: maria_vasquezv@hotmail. com

Fecha de recepción: 04-03-11

Fecha de aceptación: 06-08-12

\section{Introducción}

El tercer molar, es el último diente en erupcionar, por lo que fácilmente puede quedar impactado o sufrir desplazamientos, si no hay espacio suficiente en la arcada dentaria. Por tanto presenta una alta incidencia de inclusión que condiciona su complejidad, asimismo, la frecuencia de patología inducida por el tercer molar es muy elevada (pericoronaritis, caries en la cara distal del segundo molar o del propio tercer molar, dolor miófacial, ciertos tipos de quistes y tumores odontogénicos), debido a condiciones embriológicas y anatómicas singulares ${ }^{1}$.

Diversas fuentes consideran que la complejidad del tercer molar puede estar asociado a su relación con tejido blando (cubiertos, semicubiertos) lo mismo que con tejidos duros (cubiertos total, parcialmente o no cubiertos). ${ }^{2}$

La decisión de extracción se toma una vez realizada una correcta historia clínica, el procedimiento de la extracción puede ser simple o complicado; para poder predecir el grado de complejidad es necesario conocer los factores que le afectan; así, antes de realizar el procedimiento quirúrgico es necesario hacer una evaluación radiográfica detenida, que le permita al operador tener conocimiento de los factores que pueden dificultar el procedimiento. ${ }^{3}$ Especialmente las terceras molares inferiores debido a su morfología y posición generan un aumento del tiempo operatorio, el cual es un indicador confiable de dificultad quirúrgica. ${ }^{4}$
Respecto a la posición de la tercera molar Winter, en 1926, clasifica la posición del tercer molar en relación con el eje longitudinal del segundo molar. Las variantes son vertical, mesioangular, horizontal, distoangular, transversal e invertido 5 .

En 1933 Pell y Gregory proponen otro sistema de clasificación de acuerdo a la posición del tercer molar y a su relación respecto al segundo molar inferior y a la rama ascendente mandibular, obteniendo la profundidad relativa en el hueso. En cuanto a la primera se clasifica en Clase I, Clase II y Clase III, dependiendo del diámetro mesiodistal del tercer molar en comparación con el espacio entre la rama ascendente y la cara distal del segundo molar. En cuanto a la profundidad relativa del tercer molar en el 
hueso se divide en Posición A, Posición B y Posición C, considerando parámetros como el punto más alto de la cara oclusal del tercer molar, la cara oclusal del segundo molar inferior y la línea cervical del mismo ${ }^{6}$.

Teniendo en cuenta las consideraciones anatómicas y la posición de la pieza se obtendrán mejores resultados post operatorios. Por este motivo se han clasificado diversos factores y creado índices que ayudan a predecir la dificultad quirúrgica. 7

Para Benediktsdóttir y colaboradores, en el año 2004, las molares con dos raíces y en posición horizontal generan poco más del doble de riesgo en extender el tiempo operatorio comparado con procesos uniradiculares en posición vertical. $^{8}$

En oposición a la sobrevaloración respecto a la forma y distribución radicular, Santamaría y Arteagoitia, en 1997, consideran que un mejor indicativo de dificultad de exodoncia de las terceras molares inferiores es si estas raíces presentan un ligamento periodontal engrosado o de mayor densidad. ${ }^{9}$

La poca información en relación al tiempo quirúrgico como indicador asociado a la dificultad quirúrgica se evidencia según la literatura.

El propósito de este estudio fue evaluar los factores de dificultad (espacio disponible, profundidad, relación espacial, tamaño de folículo, integridad de hueso y mucosa y finalmente forma de las raíces) en la exodoncia de terceros molares mandibulares, relacionando con el tiempo de cirugía efectiva.

\section{Material y Método}

Se realizaron 30 extracciones de terceros molares mandibulares incluidos en pacientes que acudieron al Servicio de Cirugía Oral y Maxilofacial en el Hospital Nacional Hipólito Unanue, entre los meses enero - marzo del 2009.

Previo al procedimiento quirúrgico se tomaron radiografías panorámicas y periapical, para realizar el análisis. Para copiar la silueta de las terceras molares mandibulares incluidas, se usó el método de la observación directa ayudándonos de un negatoscopio. En todos los casos se contó con el consentimiento firmado por el paciente. Se preparo al paciente con todas las normas de asepsia y antisepsia. Se registró los datos respectivos de cada uno de los pacientes: fecha, edad, género, motivo de exodon- cia, pieza extraída. Se utilizó la técnica troncular para anestesiar la rama del dentario inferior y el nervio lingual, así como la técnica infiltrativa para el nervio bucal ${ }^{10}$. Al momento de iniciar el acto quirúrgico ya sea mediante una incisión o la realización de la sindesmotomía, se procedió a marcar con el crocasos se realizó el colgajo adecuado para la correcta visualización de la pieza. A continuación se realizó la osteotomía. Seguidamente se realizó la odontosección para conseguir la exéresis del tercer molar. Retirados todos los fragmentos y/o la pieza completa en sí, se realizó un curetaje, limpieza con agua estéril a presión. Una vez realizada la extracción dentaria se procedió a detener el cronómetro. Se consideró el momento final cuando se realizó la avulsión de la pieza. No se contabilizó el tiempo que tomo realizar la sutura. ${ }^{11}$

Para la evaluación de los resultados, se utilizó el análisis univariado, calculando para el tiempo de cirugía efectiva y la edad la media y la desviación estándar, así como sus valores mínimos y máximos. Se calculó las frecuencias para cada uno de los factores de dificultad considerados y covariables (sexo, motivo de extracción, pieza extraída). Se continúo con un análisis bivariado, en el cual se buscó relacionar el tiempo de cirugía efectiva con todas las variables cuantitativas a través del uso de coeficientes de correlación de Pearson; y medidas de asociación respecto a las variables cualitativas.

\section{Resultados}

La edad promedio de los pacientes fue de 24 años $( \pm 8,49)$, con extremos de 17 y 53 años. El 60\% estuvo por debajo del los 18 ańos, entre 18 y 25 ańos $33.3 \%$ y mayores de 25 años $6.67 \%$. nómetro el tiempo de cirugía. Según los

El tiempo promedio de cirugía efectiva fue de 9,63 minutos $( \pm 5,74)$, con mínimos de dos minutos y máximos de 23. Siendo el rango de 5 - 10 minutos el de mayor porcentaje 46,67\%), seguida del menor de 5 minutos $23,33 \%$, de 11-15 minutos $10 \%$, y de 16-20 minutos $10 \%$. (Tabla 1 )

Al relacionar el tiempo de cirugía efectiva con el motivo de consulta, género y pieza extraída no se observó relación significativa, sin embargo, la edad fue muy significativa $(\mathrm{p}=0.047)$, encontrándose una relación directa entre ambos. (Tabla 2)

Los tiempos prolongados se hallaron a medida que aumentó la edad.

Se encontró que mientras disminuía el folículo, aumentaba la integridad de hueso y mucosa y la profundidad, el tiempo de cirugía efectiva se incrementaba.

Una vez obtenidos estos datos se relacionó cada una de las variables con el factor tiempo de cirugía efectiva considerando la relación significativa con un $\mathrm{p}<0,05$. Al relacionar el tiempo de cirugía efectiva con las variables, se obtuvo que el tamaño del folículo ( $\mathrm{p}=000)$, integridad de hueso y mucosa $(\mathrm{p}=0.001)$ $y$ profundidad $(\mathrm{p}=006)$ fueron significativos. El espacio disponible, relación espacial y forma de las raíces no fueron significativas.

\section{Discusión}

En el estudio se observa que dentro de los diversos factores que influyen en el lapso de cirugía efectiva de la exodoncia del tercer molar mandibular, sólo algunos parecen tener real relevancia.

Con respecto a la ubicación, se encontró que la relación espacial más frecuente fue la mesioangular con $53,3 \%$, seguido de la posición vertical con un $26.7 \%$.

Tabla 1. Tiempos promedios de cirugía de 30 terceras molares

\begin{tabular}{ccccc}
\hline & Mínimo & Máximo & Media & Desv. Est. \\
\hline Tiempo de cirugía efectiva & 2 & 23 & 9,63 & 5,738 \\
\hline
\end{tabular}

Tabla 2. Relación entre el tiempo de cirugía efectiva y las covariables

\begin{tabular}{lc}
\hline Covariables 1/tiempo $\quad \mathrm{n} 30$ & Nivel de significancia \\
\hline Edad &, 037 \\
Género Motivo de exodoncia &, 192 \\
Motivo de exodoncia &, 198 \\
Pieza extraída &, 423 \\
\hline
\end{tabular}

*La correlación es significativa al nivel 0,05 (bilateral) 
Resultados muy similares a los encontrados por Llerena ${ }^{12}$, quien encontró en un $55 \%$ de piezas ubicadas en posición mesioangular. En un estudio realizado en el Perú Mier ${ }^{13}$ encontró sobre un total de 151 casos un 31,13 $\%$ en la posición mesioangular y 28,48 $\%$ en la posición vertical. Deza ${ }^{14}$ en su estudio encontró un 46,67\% de las piezas dentarias estudiadas en posición vertical y $20,44 \%$ en posición mesioangular. La variación en los porcentajes encontrados se pueden explicar por los cambios que ocurren en la angulación de los terceros molares, durante su proceso de erupción, así lo demuestran los estudios de Sandhu S y Kaur $\mathrm{T}^{15}$.

Con relación a los niveles de profundidad, se encontró que el nivel $\mathrm{B}$ era el más frecuente con $60 \%$ de los casos, seguido por el nivel A con $36.7 \%$ de los casos y el $3.3 \%$ nivel C. Este resultado es similar al encontrado por Castro $^{16}$. Yuasa ${ }^{17}$ hallaron que el nivel B era el más frecuente. Sin embargo Melgar ${ }^{18}$, Llerena $^{12}$ y Delgado $^{19}$ hallaron que el nivel A era el más frecuente.

$\mathrm{Al}$ observar nuestros resultados relacionados al espacio disponible, se obtuvo con mayor frecuencia la clase II en un $56.7 \%$ de los casos, para la clase I $40 \%$ y el $3.3 \%$ en la clases III. Estos datos son en cierta forma similares a lo que expresa por Delgado ${ }^{19}$, Llerena ${ }^{12}$, Mel$\operatorname{gar}^{18}$

Las formas radiculares no tuvieron asociación significativa cuando se realizó el análisis bivariado, con el tiempo de cirugía efectiva. Situación que concuerda con lo que reportaron Yuasa ${ }^{17}$, Llerena $^{12}$. Con respecto a la edad, en diversos estudios ${ }^{4,7,21-24}$, así como en el presente, es considerada como un factor significativamente importante que afecta la cirugía del tercer molar mandibular. Continuando con estas afirmaciones, Bagain ${ }^{26}$, Blondeau ${ }^{28}$, Benediktsdóttir ${ }^{29}$, chuang ${ }^{25}$, Bui ${ }^{4}$, Yuasa ${ }^{20}$ concluyen que los sucesos postoperatorios inmediatos (aumento de volumen y dolor) de una extracción de tercer molar inferior varían dependiendo de la edad del paciente, agudizándose a mayor edad.

Al relacionar todas las variables revisadas encontramos que sólo algunas de las variables fueron significativas: tamaño del folículo y la integridad de hueso y mucosa tuvieran mayor significancia, seguidos de profundidad. Renton ${ }^{7}$ y Akadiri ${ }^{28}$ hallaron que la profundidad fue el factor más importante.
Las variables espacio disponible, forma de raíces y la relación espacial no fueron significativas, a diferencia de Yuasa ${ }^{17}$ que considera aparte de profundidad, espacio disponible y el ancho de la raíz. Al relacionar el tiempo con el motivo de consulta, género y la pieza extraída no se observó diferencia significativa, mientras que se observó la existencia de una relación directamente proporcional entre tiempo y la edad, es decir a mayor edad del paciente, el tiempo seria también mucho mayor. Lo que concuerda en cierta forma con los modelos de Yuasa ${ }^{17}$.

\section{Conclusiones}

El presente trabajo demuestra que existe relación entre el tamaño del folículo, la integridad de hueso y mucosa, la profundidad y el tiempo de cirugía efectiva. También existe una relación directa entre el tiempo de cirugía efectiva y la edad; a mayor edad, mayor tiempo de cirugía efectiva. Sin embargo, el espacio disponible, la relación espacial, la forma de raíces, el motivo de exodoncia, género y pieza extraída no fueron significativas con respecto al tiempo de cirugía efectiva.

\section{Referencias Bibliográficas}

1. Gay E. Tratado de Cirugía Bucal. Tomo I. Madrid: Ergón; 2004. p. 388-457.

2. Pucara S. Relación de la complejidad de tercer molar y el tiempo de tratamiento quirúrgico [Tesis Magistral]. Lima: Universidad Cayetano Heredia; 2007.

3. Laskin D. Cirugía Bucal y Maxilofacial. Buenos aires: Editorial Médica Panamericana; 1987. p. 10-13.

4. Bui CH, Seldin EB, Dodson TB. Types, frequencies and risk factors for complications after third molar extraction. J Oral Maxillofac Surg. 2003; 61(12):1379-89.

5. Winter GB. Principles of exodontia as applied to the impacted third molar. St. Louis: American Medical Books; 1926. p. 21-58.

6. Pell GJ. Gregory BT. Impacted mandibular third molars: classification and modified techniques for removal. J Dental Digest. 1933(39):330- 338.

7. Renton T, Smeeton N, McGurk M. Factors predictive of difficulty of mandibular third molar surgery. Brit Dent J. 2001; 190(11):607-10.
8. Benediktsdóttir IS, Wenzel A, Petersen JK, Hintze H. Mandibular third molar removal: risk indicators for extended operation time, postoperative pain, and complications. Oral Surg Oral Med Oral Pathol Oral Radiol Endod. 2004; 97(4):438-46.

9. Santamaria J, Arteagoitia I. Radiologic variables of clinical significance in the extraction of impacted mandibular third molars. Oral Surg Oral Med Oral Pathol Oral Radiol Endod. 1997; 84(5):469-73.

10. Chiapasco Matteo, Cirugía Oral. Texto y atlas. Madrid: Masson; 2004. p.38-50.

11. Medeiros P. Cirugía de dientes incluidos. Extracción del Tercer Molar. Brasil: Amolca; 2006. p. 51-99.

12. Llerena G. Tiempo de cirugía efectiva en la extracción de los terceros molares realizadas por un cirujano oral y maxilofacial con experiencia [Tesis Bachiller]. Lima: Universidad Peruana Cayetano Heredia; 2006.

13. Mier H. Comparación entre el factor de dificultad con el tiempo de cirugía efectiva en la exodoncia del tercer molar inferior[Tesis Bachiller]. Lima: Universidad Peruana Cayetano Heredia; 2002.

14. Deza L. Frecuencia de las posiciones angulares más comunes de los terceros molares mandibulares [Tesis Bachiller]. Lima: Universidad Peruana Cayetano Heredia; 1999.

15. Sandhu S, Kaur T. Radiographic study of the positional changes and eruption of impacted third molars in young adults of an Asian Indian population. J Oral Maxillofac Surg. 2008; 66(8):1617-24.

16. Castro S. Impactación de terceras molares inferiores y espacio disponible para su erupción en pacientes atendidos en la Clínica Dental Del Hospital Militar Central [Tesis Bachiller]. Lima: Universidad Nacional Mayor de San Marcos; 2007.

17. Yuasa H, Kawai T, Sugiura M. Classification of surgical difficulty in extracting impacted third molars. Br J Oral Maxillofac Surg. 2002 Feb;40(1):26-31.

18. Melgar G. Tiempo efectivo de exodoncia de terceros molares inferiores relacionado con la forma de sus raíces [Tesis Bachiller]. Lima: Universidad Peruana Cayetano Heredia; 2006. 
19. Delgado B. Características de los terceros molares inferiores impactados observados en radiografías panorámicas [Tesis Bachiller]. Lima: Universidad Peruana Cayetano Heredia; 2001.

20. Yuasa H, Sugiura M. Clinical postoperative findings after removal of impacted mandibular third molars: prediction of postoperative facial swelling and pain based on preoperative variables. $\mathrm{Br} \mathrm{J}$ Oral Maxillofac Surg. 2004;42(3):20914.

21. Susarla SM, Dodson TB. Risk factors for third molar extraction difficulty. J Oral Maxillofac Surg. 2004; 62(11):1363-1371.

22. Susarla SM, Dodson TB. How well do clinicians estimate third molar extraction difficulty? J Oral Maxillofac Surg. 2005; 63(2):191-9.

23. Chuang SK, Perrott DH, Susarla SM, Dodson TB. Age as a risk factor for third molar surgery complications. J Oral Maxillofac Surg. 2007; 65(9):1685-92.

24. Chuang SK, Perrott DH, Susarla SM, Dodson TB. Risk factors for inflammatory complications following third molar surgery in adults. J Oral Maxillofac Surg. 2008; 66(11):2221-2238.

25. Gbotolorun OM, Arotiba GT, Ladeinde AL. Assessment of factors associated with surgical difficulty in impacted mandibular third molar extraction. J Oral Maxillofac Surg. 2007; 65(10):1977-83.
26. Baqain ZH, Karaky AA, Sawair F, Khaisat A, Duaibis R, Rajab LD. Frequency estimates and risk factors for postoperative morbidity after third molar removal: a prospective cohort study. J Oral Maxillofac Surg. 2008; 66(11):2276-83.

27. Blondeau F, Daniel NG. Extraction of impacted mandibular third molars: postoperative complications and their risk factors. J Can Dent Assoc. 2007; 73(4):325.

28. Akadiri OA, Obiechina AE, Arotiba JT, Fasola AO. Relative impact of patient characteristics and radiographic variables on the difficulty of removing impacted mandibular third molars. J Contemp Dent Pract. 2008;9(4):51-8. 